\title{
Plasmonic Nanoparticles as Light-Harvesting Enhancers in Perovskite Solar Cells: A User's Guide
}

\author{
S. Carretero-Palacios, A. Jiménez-Solano, and H. Míguez* \\ Institute of Materials Science of Seville, Spanish National Research Council-University of Seville, Calle Américo Vespucio 49, 41092 \\ Seville, Spain
}

Supporting Information

\begin{abstract}
In this Perspective we discuss the implications of employing metal particles of different shape, size, and composition as absorption enhancers in methylammonium lead iodide perovskite solar cells, with the aim of establishing some guidelines for the future development of plasmonic resonance-based photovoltaic devices. Hybrid perovskites present an extraordinarily high absorption coefficient which, as we show here, makes it difficult to extrapolate concepts and designs that are applied to other solution-processed photovoltaic materials. In addition, the variability of the optical constants attained from perovskite films of seemingly similar composition further complicates the analysis. We demonstrate that, by means of rigorous design, it is possible to

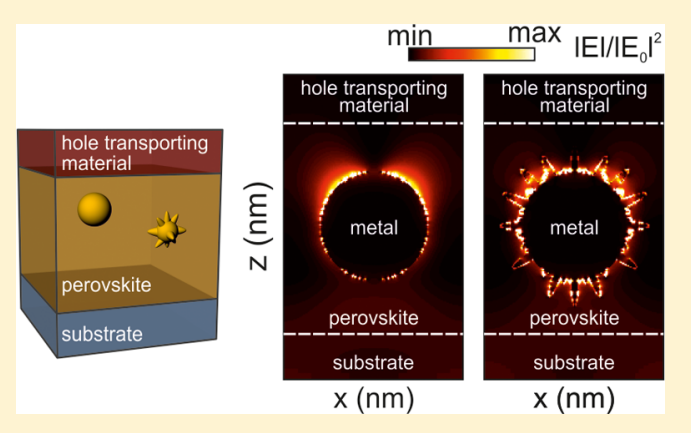
provide a realistic prediction of the magnitude of the absorption enhancement that can be reached for perovskite films embedding metal particles. On the basis of this, we foresee that localized surface plasmon effects will provide a means to attain highly efficient perovskite solar cells using films that are thinner than those usually employed, hence facilitating collection of photocarriers and significantly reducing the amount of potentially toxic lead present in the device.
\end{abstract}

$\mathrm{T}$ he use of metallic nanostructures is frequently proposed as a means to enhance the performance of photovoltaic devices. ${ }^{1-6}$ In particular, the use of metal nanoparticles and arrangements of them have been explored for almost all types of solar cells, ${ }^{7}$ be they solid-state ${ }^{8,9}$ or solutionprocessed. ${ }^{10-23}$ Perovskite solar cells (PSC), based on thin semiconductor layers with hybrid organic-metal halide composition, are not an exemption. ${ }^{24-30}$ Although these films are already efficient light harvesters, several features motivate the development of new ways to enhance even further their absorption. First, for the typical film thicknesses for which high structural quality is attained, photon capture is not as high in the range of $600 \mathrm{~nm}<\lambda<780 \mathrm{~nm}$ as it is for shorter wavelengths because the extinction coefficient rapidly decays for red frequencies. Because the number of solar photons peaks at longer visible wavelengths, this weaker red absorption affects significantly the efficiency of the incident photon-to-electron conversion process. Apart from this technical reason, there are others of an environmental nature that also justify the attempts to keep to a minimum the thickness of the perovskite layer. ${ }^{31,32}$ Indeed, the lead content of a device should be reduced as much as possible to minimize the impact of a potential leak. Also in this context, optimization of the optical design of the cell might also provide a solution by maximizing light harvesting and hence diminishing the amount of absorbing material necessary to reach a determined efficiency.
At variance with other photovoltaic devices, like silicon or dye-sensitized solar cells, the small thickness of the perovskite layers typically employed (usually $<600 \mathrm{~nm}$ ) restricts the possible modifications of their optical design that can be proposed. Although photon management schemes of different sorts have been proven to add functionality to the device, such as color or transparency, ${ }^{33,34}$ this is usually achieved at the expense of reducing its efficiency. This shortcoming results from the need to introduce significant changes in the structure of some of the cell components to attain the desired optical properties. Under these circumstances, the inclusion of metal nanoparticles, capable of supporting localized surface plasmon resonances (LSPRs), within the perovskite layer appears as a realistic means to achieve absorption enhancement altering neither the usual configuration of the cell nor the geometry of its components. Initial studies concluded that the inclusion of metal oxide $\left(\mathrm{SiO}_{2}, \mathrm{TiO}_{2}\right)$ coated metal $(\mathrm{Au}, \mathrm{Ag})$ nanoparticles (40 to $80 \mathrm{~nm}$ diameter) gave rise to a very significant photocurrent enhancement in methylammonium lead iodide (MAPI)-based devices, but interestingly, it could not be attributed to an improvement of light harvesting. ${ }^{24,25}$ Although the origin of the enhancement observed remains unknown, it

Received: May 12, 2016

Accepted: June 17, 2016

Published: June 17, 2016 
indicates that the presence of metal particles somehow favors nonradiative photocarrier generation, separation, transfer, transport, or collection. ${ }^{18,35}$ Later experimental evidence ${ }^{26-28,36}$ supported this hypothesis.

Initial observations thus raised the question as to whether perovskite film absorption can be improved by including metal particles or, on the contrary, their parasitic absorption would compensate or even surpass the enhancement they could potentially provide. To answer this question, we performed a series of calculations in which gold particles were embedded in a realistic cell configuration. ${ }^{37}$ We could demonstrate that spherical inclusions much bigger than those previously tested experimentally were required to actually improve the lightharvesting efficiency of a MAPI film. As a validation proof, our model also provided a theoretical verification of the optical absorption results reported in refs 24 and 25, as can be explicitly seen in Figure S1 in the Supporting Information. Later, further theoretical analysis by other groups confirmed this absorption enhancement considering different LSPR designs. $^{29,30}$ These works proved, at least from a fundamental perspective, that plasmonic particles can be used to enhance the absorption of perovskite films. Metal particles embedded in other parts of the cell, such as the hole-transporting or the electron-collecting (the so-called compact) layers have also been proposed, providing different mechanisms of absorption enhancement without being in contact with the perovskite. ${ }^{38}$ The increasing interest in the potentiality of this approach has been highlighted in a number of reviews, which we refer the reader to for a comprehensive account of the use of plasmonic particles in a wide range of emerging solar cell technologies. ${ }^{18,19}$

In this Perspective we evaluate the potential that the radiative effects distinctive of LSPRs of metal nanoparticles may offer to the field of perovskite photovoltaics. We extract some general conclusions that may help to identify which directions are more promising to actually realize those prospective capabilities. To do so, we start by estimating the maximum absorption enhancement achievable as a function of the perovskite film thickness considered, which serves for setting an upper limit to the magnitude of the improvement that can be expected in the best case scenario. The currently observed variability of the optical constants of MAPI thin films as a function of the preparation method employed is taken into account, and its implications in our conclusions are discussed. We then appraise the effect on the optical absorption of using embedded nanoparticles of different composition among the most standard ones, namely gold, silver, and aluminum, as well as the consequence, always in terms of the optical design, of coating them with thin dielectric shells to improve their chemical stability and durability. The influence of employing different particle geometries, particularly those that present sharp tips and hence bear the promise of a larger near-field enhancement, is also comparatively assessed. The main technical conclusion of the nonexhaustive analysis herein presented is that plasmonics may well be the key to a very significant reduction of the perovskite film thickness and hence of the amount of lead present in the device while, at the same time, ensuring an unprecedented light-harvesting efficiency. From a mechanistic perspective, our main conclusion is that the improvement predicted is neatly the result of the near optical field enhancement occurring at the longer wavelengths within the absorption band of perovskites. Our work also highlights that, in order to achieve such potential, a precise optical description and design of the materials involved is required, because most of the extracted conclusions cannot be extrapolated from what was known to work for other photovoltaic materials.

\section{Plasmonic nanoparticles may be the key to a very significant reduction of the perovskite film thickness and hence of the amount of lead present in the device while at the same time ensuring an unprecedented light-harvesting ef- ficiency.}

The diversity of methods to prepare MAPI thin films, as well as the sensitivity of their structural features to the specific synthetic conditions employed, have given rise to a wide range of compounds with apparently similar composition but very different electrical and optical properties. ${ }^{39}$ To illustrate this, some selected examples of optical constants that have been reported for perovskite thin films are provided as part of the Supporting Information (see Figure S2). ${ }^{40-43}$ To be able to extract some general conclusions, we chose a set of optical constants attained for films used in highly performing perovskite devices and that can be considered as an example of a standard in the field. ${ }^{43}$ Nevertheless, the implications of considering other perovskite films with diverse optical constants on our estimations will also receive comment throughout the text.

To set appropriate references, we first calculate the lightharvesting efficiency for the range of film thicknesses that can be found in most reports on PSC. In Figure la we show the absorptance spectra, obtained as $A_{\mathrm{p}}=1-R_{\mathrm{T}}-T_{\mathrm{T}}$, where $R_{\mathrm{T}}$ and $T_{\mathrm{T}}$ are the calculated total reflectance and the total transmittance, of a series of MAPI films of different thickness $(n$ and $k$ as in ref 43 ), while in Figure $1 \mathrm{~b}$ we depict the calculated integrated solar absorption, $\int A_{\mathrm{p}}$, which is given by

$$
\int A_{\mathrm{p}}=\int_{400}^{780} A_{\mathrm{p}}(\lambda) \cdot \operatorname{AM} 1.5 \mathrm{D}(\lambda) \mathrm{d} \lambda
$$

normalized by $\int A_{\mathrm{MAX}}$, which is the highest solar absorption achievable, i.e., that of a hypothetical infinite MAPI film capable of capturing all available sunlight photons of wavelengths within the range $400 \mathrm{~nm}<\lambda<780 \mathrm{~nm}$ (the range for which the external quantum efficiencies reported is typically not zero). We assume the films are surrounded by materials with refractive indexes similar to those of glass and an arbitrary holetransporting layers. The presence of these layers introduces certain reflectance that sets an upper limit for the achievable absorptance, to which we will refer all results of our analysis. In this case, $A_{\mathrm{p}}(\lambda)$ is the perovskite film absorptance and $\operatorname{AM} 1.5 \mathrm{D}(\lambda)$ is a standard for the solar radiance spectrum on the Earth surface assuming only direct sunlight illumination. It is interesting to note that even for the thicker films employed in actual photovoltaic devices (thickness $\approx 600 \mathrm{~nm}$ ) there is still a significant amount of nonharvested light for $\lambda>600 \mathrm{~nm}$. In actual devices, this is partially compensated by the back reflection introduced by the gold layer typically used as metal contact. ${ }^{43}$ Because shiny metallic coatings are not ${ }^{44}$ or cannot $\mathrm{be}^{45}$ used for this purpose in all cases, and in order to extract conclusions that are as general as possible, we will not assume the presence of any specific type of contact. Also, for the sake of comparison, similar estimations like those reported in Figure 1 
(a)

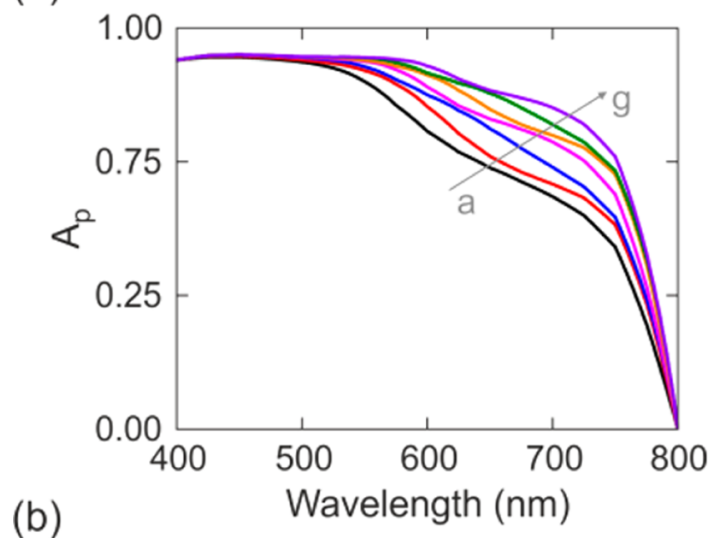

(b)

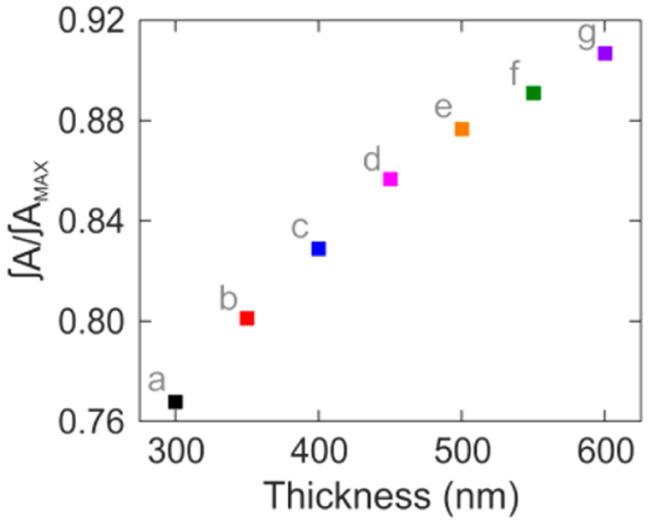

Figure 1. (a) Calculated absorptance spectra of MAPI perovskite films of different thickness. (b) Normalized solar absorption versus MAPI film thickness.

are shown in Figure S3 in the Supporting Information for a perovskite film of different optical constants, namely, like those reported in ref 41 .

Our generic description of the effect of plasmonic nanoparticles in PSC is based on a model that considers a geometry like the one described in Figure 2, in which a metal particle of

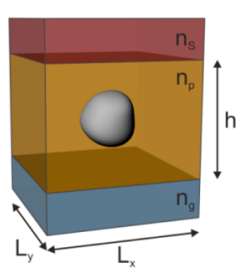

Figure 2. Schematic of the modeled perovskite unit cell, with dimensions $\left(L_{x}, L_{y}, h\right)$, embedding a metal nanoparticle. The perovskite film is assumed to be supported on glass and coated by a hole-transporting material, each layer being characterized by generic optical constants. The nanoparticle is always centered at $x=y=z=0$. Light impinges on the perovskite layer from below.

volume $v$ is located in a rectangular box made of MAPI. Full details can be found in ref 37. A commercial simulator based on the finite-difference time-domain (FDTD) method, from Lumerical Solutions Inc., was used to perform the calculations. Briefly, simulations consider a plane wave traveling along the $z$ direction and impinging on a MAPI perovskite slab of thickness $h$ containing a single metal nanoparticle. Symmetry boundary conditions are assumed along the $x$ and $y$ directions. As a consequence of the strong absorption of the matrix, no interaction between particles occurs at the concentrations herein considered. The perovskite film is surrounded by a glass substrate, of refractive index $n_{\mathrm{G}}(\omega)=1.5$, and a holetransporting material, $n_{\mathrm{S}}(\omega)=1.7$. The height of the box, $h$, equals the thickness of the film, while the lateral dimensions, $L_{x}$ and $L_{y}$, will determine the concentration or filling fraction of particles in the film:

$$
\mathrm{ff}=\frac{v}{L_{x} L_{y} h}
$$

While for the case of simple geometrical objects, such as spheres of radius $r$ and cubes of side $l, v$ is well-known $\left(v=\frac{4}{3} \pi r^{3}, v=l^{3}\right)$, a very precise description of the geometry of the particle under consideration is required when they present an arbitrary shape to determine with accuracy their volume fraction.

In Figure $3 \mathrm{a}$ we plot the normalized $\int A_{\mathrm{p}}$ attained for a perovskite film of thickness $h=300 \mathrm{~nm}$ in which metallic nanospheres of different size and composition, namely, gold (red circles), silver (green circles), and aluminum (blue circles), have been embedded. Please note that as we vary the radius of the metal particles, $r$, we increase the concentration of the inclusions because, for this illustrative case, we assume the nanoparticle is contained within a fixed volume of perovskite layer determined by $L_{x}=350 \mathrm{~nm}, L_{y}=350 \mathrm{~nm}$, and $h=300$ $\mathrm{nm}$. The values of the parameters considered determine that the particle volume concentration is approximately within the range $8 \%<\mathrm{ff}<25 \%$. For the sake of comparison, we indicate with dashed lines what would be the thickness of a MAPI film that presents a similar normalized $\int A_{\mathrm{p}}$. Although an exhaustive study will be required to determine the optimum set of parameters (size, filling fraction, exact location in the film, etc.), the first lesson that can be extracted from this data is that the three types of metal nanoparticles considered may give rise to a significant reinforcement of the integrated solar absorption of the film. In all cases, this occurs for sizes much larger than those previously tested either in perovskite or other solutionprocessed devices. ${ }^{18}$ Also, and most importantly, the results presented in Figure 3a already demonstrate the extraordinary potential of silver particles as perovskite absorption enhancers: a $300 \mathrm{~nm}$ thick MAPI film loaded with such particles absorbs as much solar radiation as one of width larger than $1 \mu \mathrm{m}$. It can be shown that the origin of the enhancement predicted lies in the localization of optical fields with $\lambda>600 \mathrm{~nm}$ within the perovskite volume closer to the particles surface. Panel b and $c$ of Figure 3 depict the calculated normalized distribution of the squared amplitude of the electric field, $|\mathbf{E}|^{2} /\left|\mathbf{E}_{0}\right|^{2}$, and the absorbed power per unit volume, $P_{\text {puv }}$, respectively, for the case of an incident plane parallel beam light of wavelength $\lambda=750$ $\mathrm{nm}$. A detailed analysis reveals that the value of the volume integrated $|\mathbf{E}|^{2} /\left|\mathbf{E}_{0}\right|^{2}$ is actually significantly higher for silver than for gold or aluminum particles. More details are given in the Supporting Information (see Figure S4). It is interesting to note that metal particles seem to behave as true light antennas when dispersed in perovskite films, this effect being the main reason for the improvement herein predicted.

To illustrate this radiative LSPR effect in more detail, we show in panels a, b, and c of Figure 4 the absorptance curves of $300 \mathrm{~nm}$ thick perovskite films containing gold, silver, and aluminum particles, respectively, with the size and concentration for which the solar integrated absorption was maximum among those presented in Figure 3a. Absorptance of both 
(a)

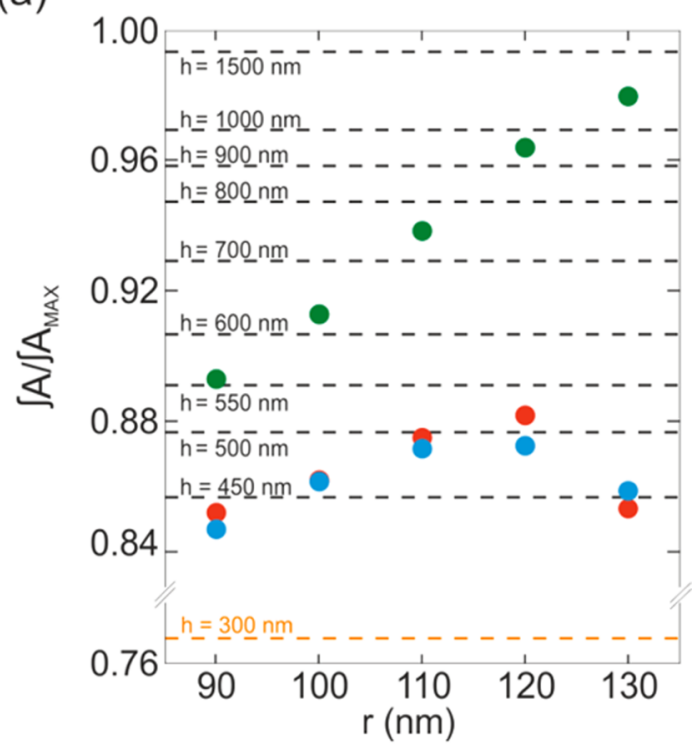

(b)

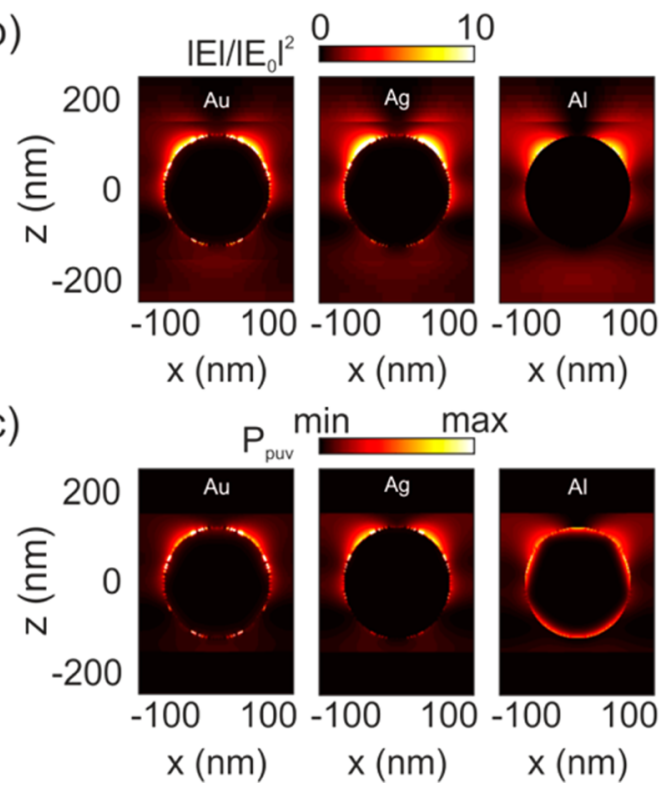

Figure 3. (a) Normalized solar absorption for a $300 \mathrm{~nm}$ MAPI film containing metal nanospheres of different radius and composition, namely, gold (red circles), silver (green circles), and aluminum (blue circles). Dashed lines indicate the normalized solar absorption of the reference perovskite film (orange line) as well as, for the sake of comparison, of thicker films. Panels $b$ and $c$ depict the spatial distribution of the normalized electric field intensity and the absorbed power per unit volume, respectively, attained at $\lambda=750 \mathrm{~nm}$ for a gold, silver, and aluminum (left, central, and right panels, respectively) spherical inclusion of radius $120 \mathrm{~nm}$.

MAPI and metal inclusions are separately evaluated using the expression

$$
A_{j}(\omega)=\omega \varepsilon_{0} \int|E(x, y, z, \omega)|^{2} n_{j}(\omega) k_{j}(\omega) \mathrm{d} V_{j}
$$

with $\omega$ the angular frequency and $E$ the electric field vector; $n_{j}(\omega)$ and $k_{j}(\omega)$ are the spectral-dependent real and imaginary parts of the refractive index of the material over whose volume, $V_{j}$, the integral is calculated. The parameter $j$ stands for either perovskite, $A_{\mathrm{p}}$, or metal, $A_{\mathrm{m}}$. It can be explicitly seen that the enhancement takes place for a wide spectral range in the red part of the absorption band of the perovskite film. On the other hand, the competing and eventually unproductive parasitic absorptance of the metal particles is provided in panels $\mathrm{d}$, e, and $\mathrm{f}$ of Figure 4 for gold, silver, and aluminum nanospheres, respectively. Contrary to what happens with other absorbing matrices, where the spectral ranges at which enhancement should occur can be guessed from the analysis of the extinction cross section of the particles in a hypothetical nonabsorbing medium that possesses a similar real part of the refractive index, ${ }^{19}$ in the case of the highly absorbing perovskite thin films such a cross section cannot be univocally defined nor approximated. This is due to the fact that the spectral and angular distribution of the scattered light intensity, as well as the amount of absorbed light, will depend strongly on the depth at which the particle is embedded in the absorbing medium. ${ }^{37}$ This behavior is radically different than that observed in, for instance, scatterers embedded in dye-sensitized titania films, where approximations like the ones described in ref 19 account well for experimental observations. ${ }^{46,47}$ Hence, to find the optimum configuration for specific perovskite solar cell designs, no shortcuts can be taken: only a full rigorous calculation in which the effect of the imaginary part of the refractive index of the matrix on the LSPR related absorption and scattering effects is considered can provide the desired outcome. However, advantageously, as can be seen in Figure 3a, the conditions to find a plasmonic configuration that improves the film absorption are not very stringent, as ample ranges of sizes and concentrations give rise to some degree of improvement. This applies also for much thicker films, in which the room for improvement is much smaller, as is shown in Figure 5. There, we plot $\int A_{\mathrm{p}} / \int A_{\mathrm{MAX}}$ versus the radius of gold particles for different concentrations, i.e., $\left(L_{x}, L_{y}\right)$ values, for the case of a $500 \mathrm{~nm}$ thick MAPI layer.

\section{The conditions to find a plasmonic configuration that improves the film absorption are not very stringent, as ample ranges of sizes and concentra- tions give rise to some degree of improvement.}

Metal nanoparticles can be synthesized not only in a wide range of compositions but also with a variety of shapes and coatings, ${ }^{48,49}$ as illustrated in Figure 6. Spheres, rods, stars, cubes, or, in general, regular and irregular polyhedrons, as well as amorphous shape particles can be attained and then coated by a layer of a different material, typically a metal oxide such as $\mathrm{SiO}_{2}, \mathrm{TiO}_{2}, \mathrm{Al}_{2} \mathrm{O}_{3}$, etc. From the point of view of their use in photovoltaics, both shape and coating influence the optical effects related to the LSPRs. In fact, the presence of sharp edges or tips is expected to favor more intense near-optical fields ${ }^{50}$ and hence give rise to higher absorption. On the other hand, a dielectric layer surrounding the metal surface might induce a strong localization of the optical field within the shell, thus diminishing the absorption of light by the solar material. However, beyond optical performance, its presence may favor the chemical stability of the metal particle either when immersed in the perovskite precursors during the synthesis or after the semiconductor matrix is formed, assuring its long-term durability. The different implications of either modifying the 


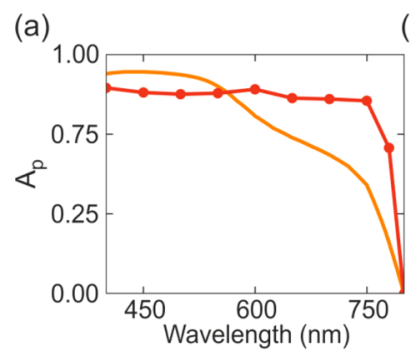

(d)

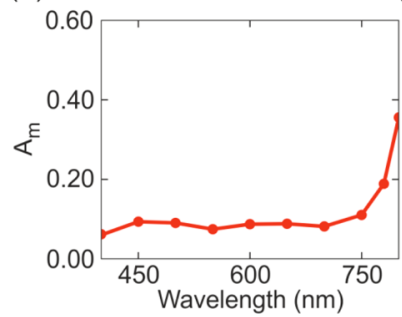

(b)

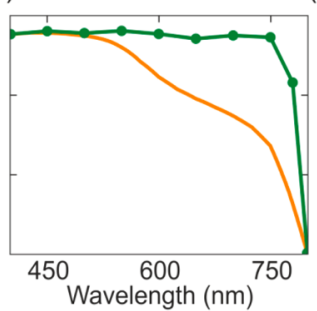

(e)

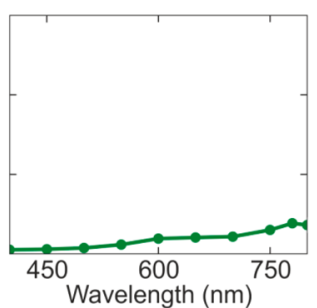

(c)

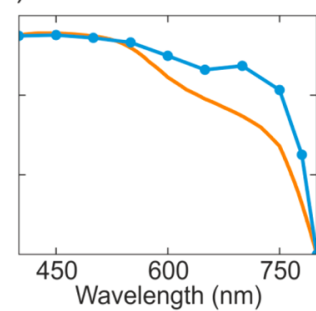

(f)

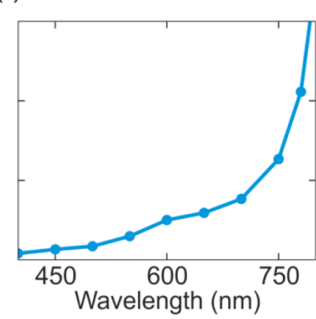

Figure 4. Perovskite absorptance spectra of selected $300 \mathrm{~nm}$ MAPI thin films containing (a) gold, (b) silver, and (c) aluminum spherical particles. Orange solid lines show the absorptance spectra of the reference perovskite film. Panels $\mathrm{d}$, e, and $\mathrm{f}$ display the total absorptance of the corresponding metal dispersions.

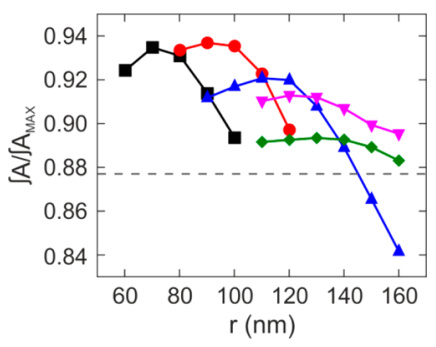

Figure 5. Normalized solar absorption for $500 \mathrm{~nm}$ thick MAPI perovskite films containing different concentrations of gold nanospheres of different sizes. Volume of evaluated unit cell is, in each case, $250 \times 250 \times 500 \mathrm{~nm}^{3}$ (black), $300 \times 300 \times 500 \mathrm{~nm}^{3}$ (red), $350 \times 350 \times 500 \mathrm{~nm}^{3}$ (blue), $450 \times 450 \times 500 \mathrm{~nm}^{3}$ (magenta), and $500 \times 500 \times 500 \mathrm{~nm}^{3}$ (green). Dashed gray line indicates the normalized solar absorption of a bare MAPI film of similar thickness.
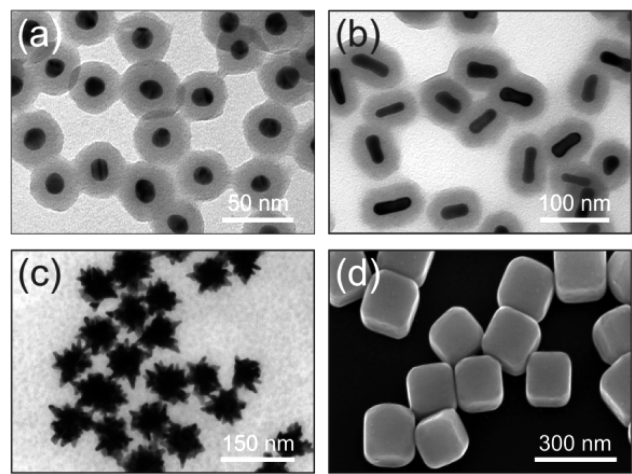

Figure 6. Illustrative examples of the wide variety of shapes in which metal nanoparticles of different composition can be synthesized: (a) silica-coated gold spheres, (b) silica-coated gold rods, (c) gold nanostars, and (d) silver cubes. Panel d reproduced with permission from ref 49. Copyright 2002 The American Association for the Advancement of Science.

morphology of the particle or introducing an outer protective layer on the radiative plasmonic effects that determine the absorption enhancement are discussed next.
Regarding shape effects, in panels a, b, and c of Figure 7, we provide a series of absorptance spectra $\left(A_{\mathrm{P}}(\lambda)\right)$ of a $500 \mathrm{~nm}$ thick MAPI film calculated considering a similar concentration of three different types of bare (uncoated) particles, namely, gold rods, gold stars, and silver cubes, respectively. In the cases of rods and cubes, the spectra shown correspond, for both $s$ and $p$ polarizations, to the average of the absorptance curves computed considering different rotation angles of the particle within the perovskite film, because the LSPR effects are highly sensitive to the relative orientation of the incident beam and the particle. ${ }^{51}$ In all cases, the spectra of both a homogeneous MAPI film (orange curve) and the same film containing gold nanospheres (red curve) is plotted for the sake of comparison. The volume occupied by the metal inclusions is also similar in all films. Contrary to what could be expected, the effect of sharper features, around which light efficiently localizes, as can be seen in Figure $7 d$, is compensated by poorer optical field concentration at other regions of the particle surface, overall providing an absorption enhancement comparable to the one attained from round particles (Figure $7 \mathrm{a}-\mathrm{c}$ ). This can be readily seen in the case of the cubes (Figure $7 \mathrm{~d}$, last panel), where the electric field concentrates significantly only around the vertices. A more thorough description of this compensating effect in the case of nanostars is given in the Supporting Information (Figure S5). The case of the rods deserves particular attention because the absorption enhancement observed is strongly dependent on the orientation of their long axis with respect to the incident beam. Indeed, if it was possible to have all rods vertically oriented (i.e., parallel to the incident beam), the absorption obtained would significantly overcome that of a dispersion of the same volume of spherical particles, as described in Figure S6. However, the average of the spectra corresponding to different relative alignments results in an enhancement factor similar to that achieved with spheres. Note also that rods of the same volume but different aspect ratio would provide dissimilar outcome.

Finally, the consequences of shielding the metal particles with dielectric coatings are also assessed. In Figure 8a we show the normalized solar integrated absorption of a series of MAPI films containing gold spheres covered by silica shells of different 
(a)

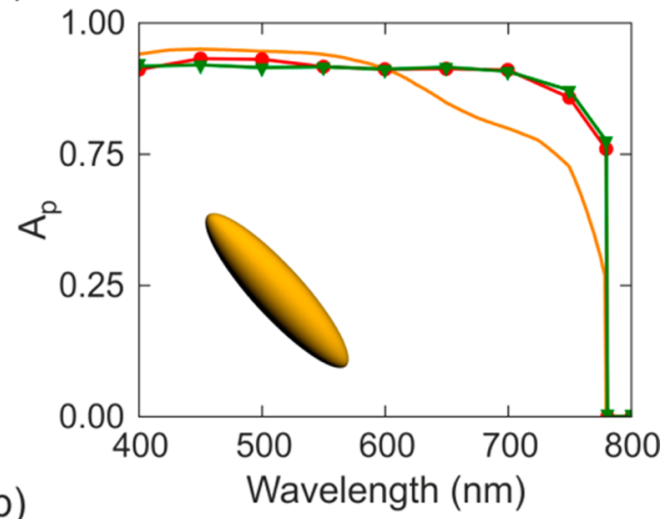

(b)

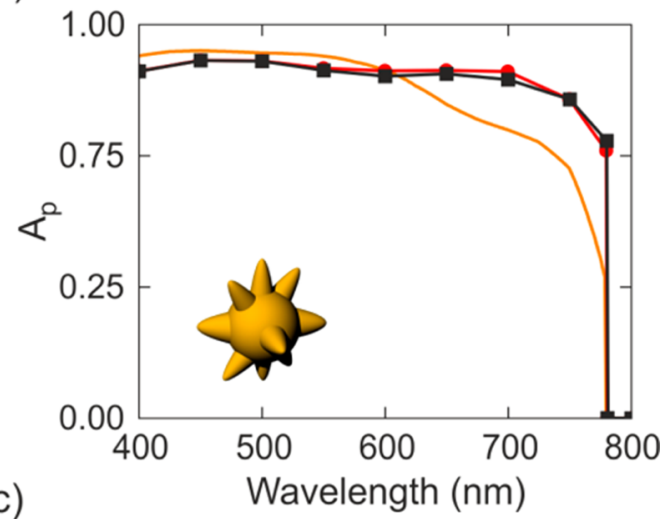

c)

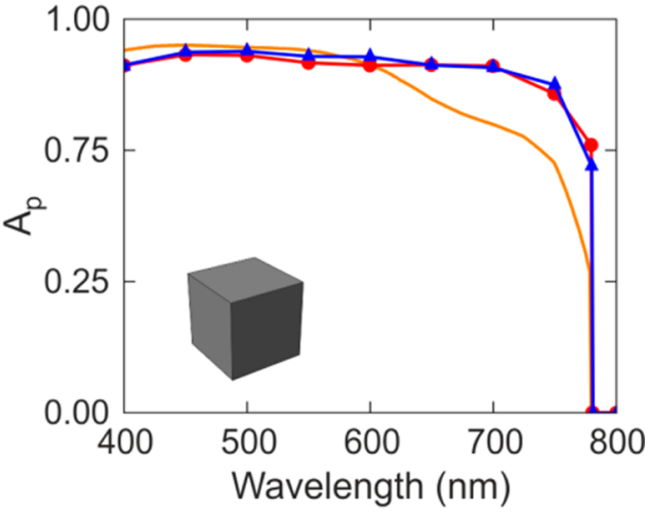

(d)

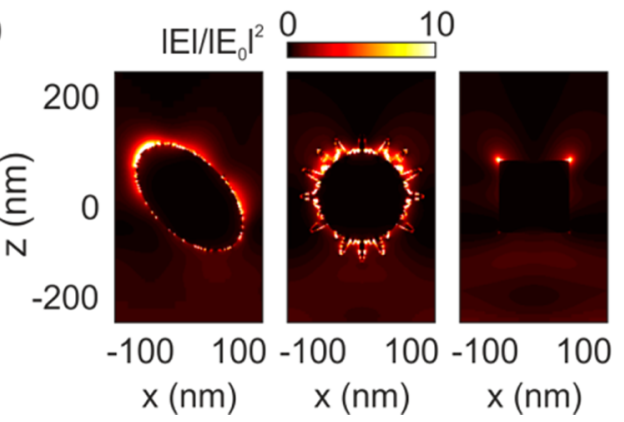

Figure 7. Absorptance of a $300 \times 300 \times 500 \mathrm{~nm}^{3}$ MAPI perovskite film embedding (a) gold nanorods (of aspect ratio 1.73), (b) gold nanostars, and (c) silver nanocubes, all of them with the same volume as that of a $90 \mathrm{~nm}$ radius sphere. In all cases, random orientation of the nanoparticles dispersed in the perovskite slab, for $s$ and $p$ polarizations, are assumed. The absorptance spectra of a homogeneous MAPI film of the same thickness without (orange line) and with (red line) $90 \mathrm{~nm}$ radius spheres embedded are also plotted for the sake of comparison. The contour plot in panel d
Figure 7. continued

represents the spatial distribution of the normalized electric field intensity at $\lambda=750 \mathrm{~nm}$.

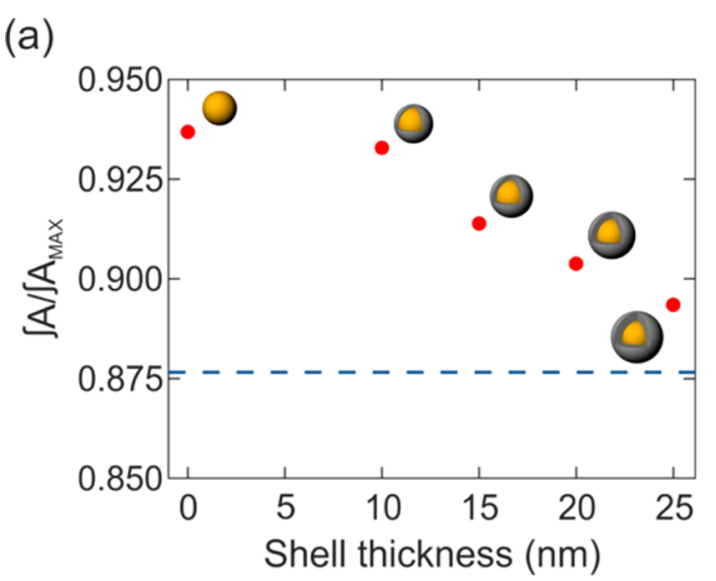

(b)

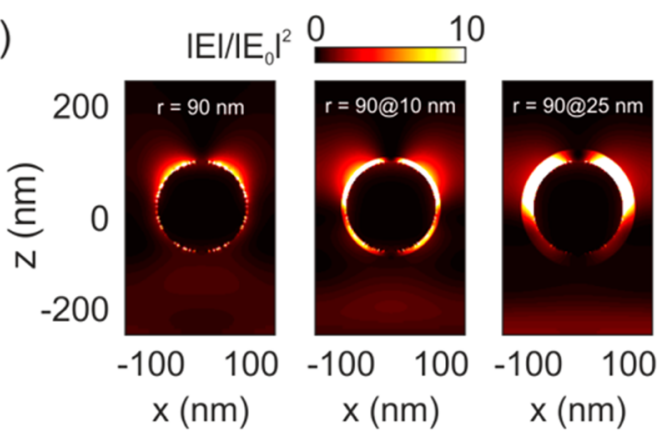

(c)

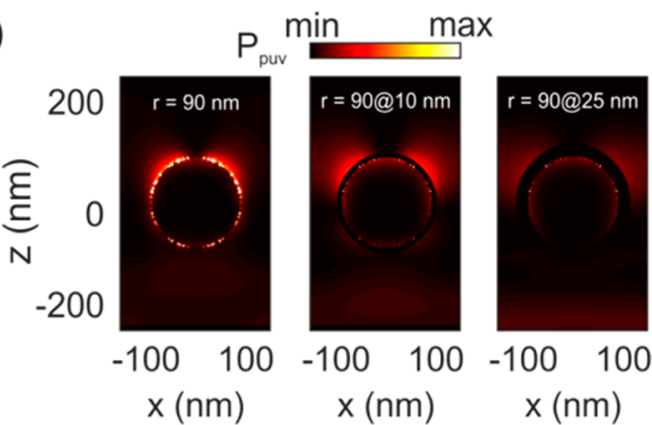

Figure 8. (a) Normalized solar absorption of a $500 \mathrm{~nm}$ MAPI film embedding $90 \mathrm{~nm}$ radius gold spheres as a function of the thickness of the protective silica shell considered, as schematized in the insets. The blue dashed line is placed at the normalized solar absorption value of a reference film of similar thickness but without embedded particles. Panels $b$ and $c$ depict the spatial distribution of the normalized electric field intensity and the absorbed power per unit volume, respectively, attained at $\lambda=750 \mathrm{~nm}$.

thickness $\left(\mathrm{Au} @ \mathrm{SiO}_{2}\right)$. It can be seen that, although the overall light harvesting by the perovskite film is always larger when uncoated particles are used, the presence of a dielectric protective layer does not necessarily prompt the collapse of the enhancement effect of the metal inclusions, as long as its thickness is not too big. More precisely, for the case chosen as example, a $10 \mathrm{~nm}$ wide silica layer allows preserving a great deal of the reinforcement effect while potentially providing chemical stability to the particle. Analysis of the spatial distribution of the calculated optical field, $|\mathbf{E}|^{2} /\left|\mathbf{E}_{0}\right|^{2}$, and the absorbed power per unit volume, $P_{\text {puv }}$, reveals that the addition of a dielectric 
protective layer to the metal particle causes a strong localization of the optical fields in the shell, in which no absorption takes place. This can be readily seen by comparing panels $b$ and $c$ of Figure 8. However, for the thinner layers considered, this effect is compensated by a larger scattering, which allows maintaining a high enhancement. Also, $\int A_{\mathrm{p}} / \int A_{\mathrm{MAX}}$ rapidly approaches the efficiency of the reference film as the thickness of the shell is increased. Thicker shells than those presented in Figure 8a should eventually have a deleterious effect on the film productive absorption. Further details, such as the analysis of different combinations of gold particle size and shell thickness, can be found in the Supporting Information (Figure S7).

In this Perspective, by means of theoretical modeling and calculations, we realize an illustrated overview of the role metal nanoparticles can play in perovskite-based photovoltaic devices. Our focus is exclusively on the radiative effects associated with the localized surface plasmon resonance characteristic of metal nanoparticles. We have tried to consider material configurations and optical constants as realistic as possible, establishing a set of references without forgetting the evident problem of standardization that exists in the field and employing metal nanoparticles of different composition, size, shape, and internal structure.

Our main conclusion if that metal nanoparticle inclusions do behave as highly efficient absorption enhancers that may allow maximizing the light-harvesting properties of a perovskite film of a given thickness. This should result not only in an optimization of the optical performance of the device but also in a reduction of the amount of absorbing material employed, hence reducing the environmental impact of the use of leadbased semiconductors like the hybrid perovskites herein considered. Our analysis demonstrates that, in all cases, both metal particle sizes and concentrations of choice are well-above those employed for other photovoltaic devices, a consequence of the extraordinarily high absorption coefficient of the perovskite films. Interestingly, the range of parameters of the solid dispersion for which enhancement is observed is not restrictive. In addition, a series of remarkable features, such as the enormous potential of silver nanoparticles, which provide the largest enhancements among all metals considered, or the possibility to protect the particle surface with a dielectric coating while preserving the sought-after absorption enhancement effects, serve to point out promising future directions of research.

\section{Absorption-enhancing effects of even larger magnitude should be expected for other hybrid perovskites of rele- vance in photovoltaics.}

The reader should be aware that the results herein presented constitute by no means an exhaustive analysis of all possible designs incorporating metal nanoparticles; a thorough optimization process should be carried out for each specific case under consideration. Although all examples herein chosen deal with methylammonium lead iodide, absorption-enhancing effects of even larger magnitude should be expected for other hybrid perovskites of relevance in photovoltaics in which other halide, metal, or organic ions are present. Also, the effect of metal particles on the charge transport through the perovskite film has not been accounted for, nor the potential benefit of nonradiative effects such as hot electron transfer and plasmon- induced resonant energy transfer. On the basis of the encouraging perspectives herein presented, we foresee that an integral study of all radiative and nonradiative plasmonic effects of metal particles embedded in perovskite films might allow finding configurations that yield an even better performance while further reducing the amount of photovoltaic material employed.

\section{ASSOCIATED CONTENT}

\section{S Supporting Information}

The Supporting Information is available free of charge on the ACS Publications website at DOI: 10.1021/acsenergylett.6b00138.

Validation of the model employed, optical constants for different MAPI thin films, effect of the metal particle composition and shape on the near optical field intensity distribution, details of the calculations at different orientation of rods and cubes, analysis of the effect of the shell thickness for $\mathrm{Au} @ \mathrm{SiO}_{2}$ nanospheres, and the Tauc plot of the modeled perovskite (PDF)

\section{AUTHOR INFORMATION}

\section{Corresponding Author}

*E-mail: h.miguez@csic.es.

\section{Notes}

The authors declare no competing financial interest.

\section{Biographies}

Sol Carretero-Palacios received her Ph.D. at the University of Zaragoza in 2011. She worked as a postdoc in the LMU-München University until 2013. Since 2014, she has been working as a postdoctoral researcher in the Multifunctional Optical Materials (MOM) group.

Alberto Jiménez-Solano obtained his Master degree in Materials Science at University of Sevilla (US, Spain) in 2012. He has been a $\mathrm{Ph} . \mathrm{D}$. student in the MOM group since 2012 where he focused on the absorption and emission properties of nanomaterials integrated in photonics structures.

Prof. Hernán Míguez received his $\mathrm{PhD}$ at the Autonomous University of Madrid in 2000. Since 2004 he holds a permanent position in the Spanish National Research Council (CSIC), where he works as a Research Professor in the Institute of Materials Science of Seville leading the group of Multifunctional Optical Materials.

\section{ACKNOWLEDGMENTS}

Financial support of the European Research Council under the European Union's Seventh Framework Programme (FP7/ 2007-2013)/ERC Grant Agreement no. 307081 (POLIGHT) and the Spanish Ministry of Economy and Competitiveness under grant MAT2011-23593 and MAT2014-54852-R, is gratefully acknowledged. A.J.-S. acknowledges the Spanish Ministry of Economy and Competitiveness for funding through an FPI program under the project MAT2011-23593. We are grateful to Miguel Anaya for his critical reading of the manuscript.

\section{REFERENCES}

(1) Atwater, H. A.; Polman, A. Plasmonics for Improved Photovoltaic Devices. Nat. Mater. 2010, 9, 205-213.

(2) Schuller, J. A.; Barnard, E. S.; Cai, W.; Jun, Y. C.; White, J. S.; Brongersma, M. L. Plasmonics for Extreme Light Concentration and Manipulation. Nat. Mater. 2010, 9, 193-204. 
(3) Kume, T.; Hayashi, S.; Ohkuma, H.; Yamamoto, K. Enhancement of Photoelectric Conversion Efficiency in Copper Phthalocyanine Solar Cell: White Light Excitation of Surface Plasmon Polaritons. Jpn. J. Appl. Phys. 1995, 34, 6448-6451.

(4) Stenzel, O.; Stendal, A.; Voigtsberger, K.; von Borczyskowski, C. Enhancement of the Photovoltaic Conversion Efficiency of Copper Phthalocyanine Thin Film Devices by Incorporation of Metal Clusters. Sol. Energy Mater. Sol. Cells 1995, 37, 337-348.

(5) Ferry, V. E.; Munday, J. N.; Atwater, H. A. Design Considerations for Plasmonic Photovoltaics. Adv. Mater. 2010, 22, 4794-4808.

(6) Pala, R. A.; White, J.; Barnard, E.; Liu, J.; Brongersma, M. L. Design of Plasmonic Thin-Film Solar Cells with Broadband Absorption Enhancements. Adv. Mater. 2009, 21, 3504-3509.

(7) de Aberasturi, D. J.; Serrano-Montes, A. B.; Liz-Marzán, L. M. Modern Applications of Plasmonic Nanoparticles: From Energy to Health. Adv. Opt. Mater. 2015, 3, 602-617.

(8) Catchpole, K. R.; Polman, A. Design Principles for Particle Plasmon Enhanced Solar Cells. Appl. Phys. Lett. 2008, 93, 191113.

(9) Pryce, I. M.; Koleske, D. D.; Fischer, A. J.; Atwater, H. A. Plasmonic Nanoparticle Enhanced Photocurrent in GaN/InGaN/GaN Quantum Well Solar Cells. Appl. Phys. Lett. 2010, 96, 153501.

(10) Wu, J.-L.; Chen, F.-C.; Hsiao, Y.-S.; Chien, F.-C.; Chen, P.; Kuo, C.-H.; Huang, M. H.; Hsu, C.-S. Surface Plasmonic Effects of Metallic Nanoparticles on the Performance of Polymer Bulk Heterojunction Solar Cells. ACS Nano 2011, 5, 959-967.

(11) Yang, X.; Chueh, C.-C.; Li, C.-Z.; Yip, H.-L.; Yin, P.; Chen, H.; Chen, W.-C.; Jen, A. K.-Y. High-Efficiency Polymer Solar Cells Achieved by Doping Plasmonic Metallic Nanoparticles into Dual Charge Selecting Interfacial Layers to Enhance Light Trapping. Adv. Energy Mater. 2013, 3, 666-673.

(12) Gan, Q.; Bartoli, F. J.; Kafafi, Z. H. Plasmonic-Enhanced Organic Photovoltaics: Breaking the $10 \%$ Efficiency Barrier. Adv. Mater. 2013, 25, 2385-2396.

(13) Standridge, S. D.; Schatz, G. C.; Hupp, J. T. Distance Dependence of Plasmon-Enhanced Photocurrent in Dye-Sensitized Solar Cells. J. Am. Chem. Soc. 2009, 131, 8407-8409.

(14) Brown, M. D.; Suteewong, T.; Kumar, R. S. S.; D’Innocenzo, V.; Petrozza, A.; Lee, M. M.; Wiesner, U.; Snaith, H. J. Plasmonic DyeSensitized Solar Cells Using Core-Shell Metal-Insulator Nanoparticles. Nano Lett. 2011, 11, 438-445.

(15) Choi, H.; Chen, W. T.; Kamat, P. V. Know Thy Nano Neighbor: Plasmonic versus Electron Charging Effects of Metal Nanoparticles in Dye-Sensitized Solar Cells. ACS Nano 2012, 6, 4418-4427.

(16) Kawawaki, T.; Tatsuma, T. Enhancement of PbS Quantum DotSensitized Photocurrents Using Plasmonic Gold Nanoparticles. Phys. Chem. Chem. Phys. 2013, 15, 20247-20251.

(17) Kawawaki, T.; Wang, H.; Kubo, T.; Saito, K.; Nakazaki, J.; Segawa, H.; Tatsuma, T. Efficiency. Enhancement of PbS Quantum Dot/ZnO Nanowire Bulk-Heterojunction Solar Cells by Plasmonic Silver Nanocubes. ACS Nano 2015, 9, 4165-4172.

(18) Erwin, W. R.; Zarick, H. F.; Talbert, E. M.; Bardhan, R. Light Trapping in Mesoporous Solar Cells with Plasmonic Nanostructures. Energy Environ. Sci. 2016, 9, 1577-1601.

(19) Arinze, E. S.; Qiu, B.; Nyirjesy, G.; Thon, S. M. Plasmonic Nanoparticle Enhancement of Solution-Processed Solar Cells: Practical Limits and Opportunities. ACS Photonics 2016, 3, 158-173.

(20) Hsiao, Y.-S.; Charan, S.; Wu, F.-Y.; Chien, F.-C.; Chu, C.-W.; Chen, P.; Chen, F.-C. Improving the Light Trapping Efficiency of Plasmonic Polymer Solar Cells through Photon Management. J. Phys. Chem. C 2012, 116, 20731-20737.

(21) Choi, H.; Lee, J.-P.; Ko, S.-J.; Jung, J.-W.; Park, H.; Yoo, S.; Park, O.; Jeong, J.-R.; Park, S.; Kim, J. Y. Multipositional Silica-Coated Silver Nanoparticles for High-Performance Polymer Solar Cells. Nano Lett. 2013, 13, 2204-2208.

(22) Chuang, M.-K.; Lin, S.-W.; Chen, F.-C.; Chu, C.-W.; Hsu, C.-S. Gold Nanoparticle-Decorated Graphene Oxides for PlasmonicEnhanced Polymer Photovoltaic Device. Nanoscale 2014, 6, 15731579.
(23) Chou, C.-H.; Chen, F.-C. Plasmonic Nanostructures for Light Trapping in Organic Photovoltaic Devices. Nanoscale 2014, 6, 84448458.

(24) Zhang, W.; Saliba, M.; Stranks, S. D.; Sun, Y.; Shi, X.; Wiesner, U.; Snaith, H. J. Enhancement of Perovskite-Based Solar Cells Employing Core-Shell Metal Nanoparticles. Nano Lett. 2013, 13, 4505-4510.

(25) Saliba, M.; Zhang, W.; Burlakov, V. M.; Stranks, S. D.; Sun, Y.; Ball, J. M.; Johnston, M. B.; Goriely, A.; Wiesner, U.; Snaith, H. J. Plasmonic-Induced Photon Recycling in Metal Halide Perovskite Solar Cells. Adv. Funct. Mater. 2015, 25, 5038-5046.

(26) Lu, Z.; Pan, X.; Ma, Y.; Li, Y.; Zheng, L.; Zhang, D.; Xu, Q.; Chen, Z.; Wang, S.; Qu, B.; et al. Plasmonic-Enhanced Perovskite Solar Cells Using Alloy Popcorn Nanoparticles. RSC Adv. 2015, 5, 11175-11179.

(27) Mali, S. S.; Shim, C. S.; Kim, H.; Patil, P. S.; Hong, C. K. In Situ Processed Gold Nanoparticle-Embedded TiO2 Nanofibers Enabling Plasmonic Perovskite Solar Cells to Exceed 14\% Conversion Efficiency. Nanoscale 2016, 8, 2664-2677.

(28) Lee, D. S.; Kim, W.; Cha, B. G.; Kwon, J.; Kim, S. J.; Kim, M.; Kim, J.; Wang, D. H.; Park, J. H. Self-Position of Au NPs in Perovskite Solar Cells: Optical and Electrical Contribution. ACS Appl. Mater. Interfaces 2016, 8, 449-454.

(29) Cai, B.; Peng, Y.; Cheng, Y. B.; Gu, M. 4-Fold Photocurrent Enhancement in Ultrathin Nanoplasmonic Perovskite Solar Cells. Opt. Express 2015, 23, A1700-A1706.

(30) Yue, L.; Yan, B.; Attridge, M.; Wang, Z. Light Absorption in Perovskite Solar Cell: Fundamentals and Plasmonic Enhancement of Infrared Band Absorption. Sol. Energy 2016, 124, 143-152.

(31) Babayigit, A.; Ethirajan, A.; Muller, M.; Conings, B. Toxicity of Organometal Halide Perovskite Solar Cells. Nat. Mater. 2016, 15, $247-251$.

(32) Babayigit, A.; Thanh, D. D.; Ethirajan, A.; Manca, J.; Muller, M.; Boyen, H.-G.; Conings, B. Assessing the Toxicity of Pb- and Sn-based Perovskite Solar Cells in Model Organism Danio rerio. Sci. Rep. 2016, 6, 18721.

(33) Zhang, W.; Anaya, M.; Lozano, G.; Calvo, M. E.; Johnston, M. B.; Míguez, H.; Snaith, H. J. Highly Efficient Perovskite Solar Cells with Tunable Structural Color. Nano Lett. 2015, 15, 1698-1702.

(34) Roldan-Carmona, C.; Malinkiewicz, O.; Betancur, R.; Longo, G.; Momblona, C.; Jaramillo, F.; Camacho, L.; Bolink, H. High Efficiency Single-Junction Semitransparent Perovskite Solar Cells. Energy Environ. Sci. 2014, 7, 2968-2973.

(35) Li, J.; Cushing, S. K.; Meng, F.; Senty, T. R.; Bristow, A. D.; Wu, N. Plasmon-Induced Resonance Energy Transfer for Solar Energy Conversion. Nat. Photonics 2015, 9, 601-607.

(36) Yuan, Z. C.; Wu, Z. W.; Bai, S.; Xia, Z. H.; Xu, W. D.; Song, T.; Wu, H. H.; Xu, L. H.; Si, J. J.; Jin, Y. Z.; et al. Hot-Electron Injection in a Sandwiched TiOx-Au-TiOx Structure for High-Performance Planar Perovskite Solar Cells. Adv. Energy Mater. 2015, 5, 1500038.

(37) Carretero-Palacios, S.; Calvo, M. E.; Míguez, H. Absorption Enhancement in Organic-Inorganic Halide Perovskite Films with Embedded Plasmonic Gold Nanoparticles. J. Phys. Chem. C 2015, 119, 18635-18640.

(38) Hsu, H.-L.; Juang, T.-Y.; Chen, C.-P.; Hsieh, C.-M.; Yang, C.-C.; Huang, C.-L.; Jeng, R.-J. Enhanced Efficiency of Organic and Perovskite Photovoltaics from Shape-Dependent Broadband Plasmonic Effects of Silver Nanoplates. Sol. Energy Mater. Sol. Cells 2015, 140, 224-231.

(39) Green, M. A.; Jiang, Y.; Soufiani, A. M.; Ho-Baillie, A. Optical Properties of Photovoltaic Organic-Inorganic Lead Halide Perovskites. J. Phys. Chem. Lett. 2015, 6, 4774-4785.

(40) De Wolf, S.; Holovsky, J.; Moon, S.-J.; Löper, P.; Niesen, B.; Ledinsky, M.; Haug, F.-J.; Yum, J.-H.; Ballif, C. Organometallic Halide Perovskites: Sharp Optical Absorption Edge and its Relation to Photovoltaic Performance. J. Phys. Chem. Lett. 2014, 5, 1035-1039.

(41) Anaya, M.; Lozano, G.; Calvo, M. E.; Zhang, W.; Johnston, M. B.; Snaith, H. J.; Miguez, H. Optical Description of Mesostructured 
Organic-Inorganic Halide Perovskite Solar Cells. J. Phys. Chem. Lett.

2015, 6, 48-53.

(42) Lin, Q.; Armin, A.; Nagiri, R. C. R.; Burn, P. L.; Meredith, P. Electro-Optics of Perovskite Solar Cells. Nat. Photonics 2015, 9, 106112.

(43) Correa-Baena, J. P.; Anaya, M.; Lozano, G.; Tress, W.; Domanski, K.; Saliba, M.; Matsui, T.; Jacobsson, T. J.; Calvo, M. E.; Abate, A.; et al. A. Unbroken Perovskite: Interplay of Morphology, Electro-optical Properties, and Ionic Movement. Adv. Mater. 2016, DOI: 10.1002/adma.201600624.

(44) Mei, A.; Li, X.; Liu, L.; Ku, Z.; Liu, T.; Rong, Y.; Xu, M.; Hu, M.; Chen, J.; Yang, Y.; et al. A hole-conductor-free, fully printable mesoscopic perovskite solar cell with high stability. Science 2014, 345, 295-298.

(45) Albrecht, S.; Saliba, M.; Correa-Baena, J. P.; Lang, F.; Kegelmann, L.; Mews, M.; Steier, L.; Abate, A.; Rappich, J.; Korte, L.; et al. Monolithic Perovskite/Silicon-Heterojunction Tandem Solar Cells Processed at Low temperature. Energy Environ. Sci. 2016, 9, 8188.

(46) Gálvez, F. E.; Barnes, P.; Halme, J.; Míguez, H. Dye Solar Cells as Optically Random Photovoltaic Media. Energy Environ. Sci. 2014, 7, 689-697.

(47) Miranda-Muñoz, J. M.; Carretero-Palacios, S.; Jiménez-Solano, A.; Li, Y.; Lozano, G.; Míguez, H. Efficient bifacial dye-sensitized solar cells through disorder by design. J. Mater. Chem. A 2016, 4, 19531961.

(48) Barbosa, S.; Agrawal, A.; Rodríguez-Lorenzo, L.; PastorizaSantos, I.; Alvarez-Puebla, R. A.; Kornowski, A.; Weller, H.; LizMarzán, L. M. Tuning Size and Sensing Properties in Colloidal Gold Nanostars. Langmuir 2010, 26, 14943-14950.

(49) Sun, Y.; Xia, Y. N. Shape-Controlled Synthesis of Gold and Silver Nanoparticles. Science 2002, 298, 2176-2179.

(50) Nelayah, J.; Kociak, M.; Stéphan, O.; García de Abajo, F. J.; Tencé, M.; Henrard, L.; Taverna, D.; Pastoriza-Santos, I.; Liz-Marzán, L. M.; Colliex, C. Mapping surface plasmons on a single metallic nanoparticle. Nat. Phys. 2007, 3, 348-353.

(51) Khlebtsov, B. N.; Khlebtsov, N. G. Multipole plasmons in metal nanorods: Scaling properties and dependence on particle size, shape, orientation, and dielectric environment. J. Phys. Chem. C 2007, 111, 11516-11527. 